\title{
Science behind nacre: matrix-directed mineralization at ambient condition
}

\author{
Qunfeng Cheng ${ }^{1}$ and Lei Jiang ${ }^{1,2^{*}}$
}

Mother Nature has demonstrated the importance of structural designs at multiscale: biological structural materials frequently adopt complex hierarchical structures to optimize their mechanical performance that is far beyond their abiotic counterparts [1]. One of the most studied biological materials is the nacreous part in some mollusk shells, i.e., nacre, due to its amazing 'brick-and-mortar' structure. Although nacre is composed of about $95 v . \%$ of aragonite and $5 v . \%$ of organic matrix [2], its toughness is three thousand times higher than that of pure aragonite through both intrinsic and extrinsic toughening mechanisms while maintaining a high strength [3]. Plenty of observations have uncovered the basic processes of nacre growth as the following: the formation of several layers of organic matrix mainly composed of insoluble $\beta$-chitin, the attachment growth of aragonite platelets in the presence of proteins and inorganic ions through channels in the organic layers (involving the aggregation and crystallization of precursor, the occlusion of organic moieties, etc.), and the lateral growth of nascent platelets to form a Voronoi pattern [2].

Bio-inspired structural materials have witnessed significant progress. Inspired by the structure of nacre, composite films have been fabricated via various techniques such as layer-by-layer assembly or spin-coating method [4-6]. At the same time, an effective way to make three dimensional ceramic composites has also been reported based on freeze casting technique [7-10]. Briefly, the ceramic particles in the slurry are separated by growing ice crystals induced by directional cooling to form alternating ceramic/ice layers. After the removal of ice via vacuum drying, the remaining ceramic laminates are sintered at a high temperature (> $1000^{\circ} \mathrm{C}$ ) to ensure the coalescence of the ceramic particles.
Alternatively, by taking advantage of magnetic field-assisted alignment, heterogeneous composites that mainly consist of $\mathrm{Fe}_{3} \mathrm{O}_{4}$-magnetized alumina platelets have been fabricated [11]. Besides the high flexural strength and fracture toughness, these textured ceramic composites produced by different techniques undergo large strains before failure, which is rarely seen for traditional ceramics.

Despite of the attractive properties of these artificial nacre-like materials, there are some drawbacks that impede their applications. For instance, previous techniques are slurry-based and a sintering step is inevitable, and thus they are not available for numerous materials. Moreover, previously reported nacre-like composites did not possess completely replicated natural nacre's fine features such as platelet waviness and dovetail structure, which were also the significant designs of natural nacre to enhance its mechanical performance.

Recently, a biomimetic materials research group led by Prof. Shu-Hong Yu from the University of Science and Technology of China (USTC) developed a novel mesoscale "assembly-and-mineralization" approach for the fabrication of synthetic nacre by precisely mimicking the process of mineralization of nacre [12] (Fig. 1a). Based on freeze casting, the researchers firstly demonstrated the scaffold with a predesigned laminated chitin matrix, which provided space for the mineralization of $\mathrm{CaCO}_{3}$ under mild conditions in a circular system, mimicking the real biomineralization process in mollusks. The mineralized matrix is infiltrated with silk fibroin and then hot-pressed, resulting in an exact artificial nacre until now. This synthetic nacre bears a striking resemblance to natural nacre with respect to the components ( 90 wt.\% of $\mathrm{CaCO}_{3}$ and $\sim 10$ wt.\%

\footnotetext{
${ }^{1}$ Key Laboratory of Bio-Inspired Smart Interfacial Science and Technology of Ministry of Education, School of Chemistry and Environment, Beihang University, Beijing 100191, China

${ }^{2}$ Laboratory of Bio-inspired Smart Interface Science, Technical Institute of Physics and Chemistry, Chinese Academy of Science, Beijing 100191, China

*Email: jianglei@iccas.ac.cn
} 
of chitin and silk fibroin), and contains the hierarchical structures and the fine features (waviness, roughness and dovetail) (Fig. 1b). On account of the 'brick-and-mortar' structure of the synthetic nacre, its flexural strength and fracture toughness are comparable to that of natural nacre. Specifically, the synthetic nacre also exhibits good crack-resistance property (Fig. 1c).

In fact, the nacre is a typical binary cooperative complementary material system [13], showing hard inorganic component of calcium carbonate and soft organic component of protein matrix assembled via a "brick-and-mortar" hierarchical micro/nano-scale structure (Fig. 2). In 2006,
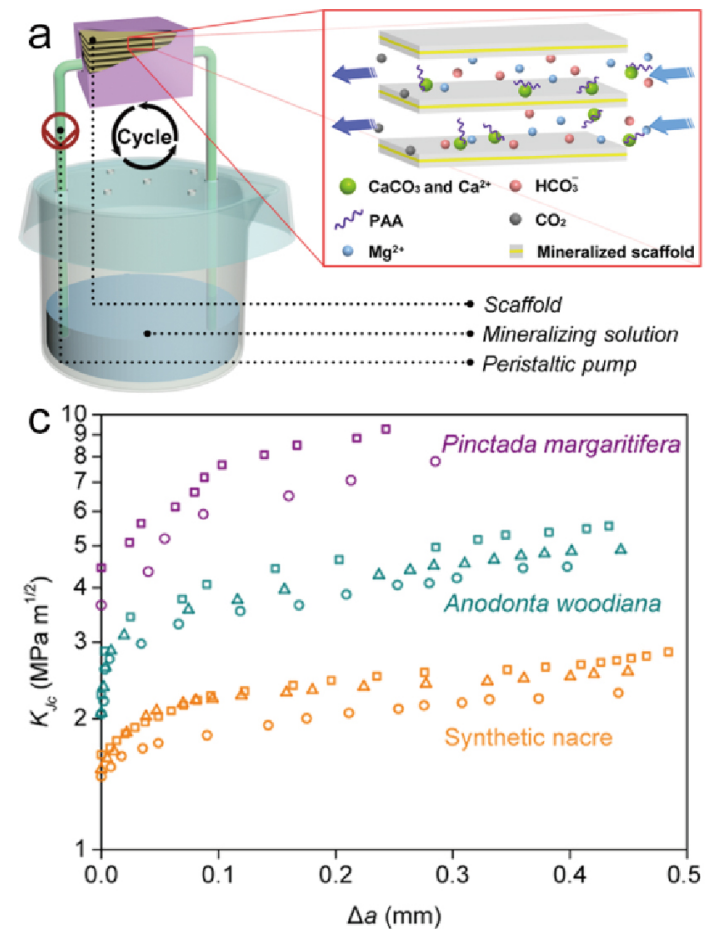
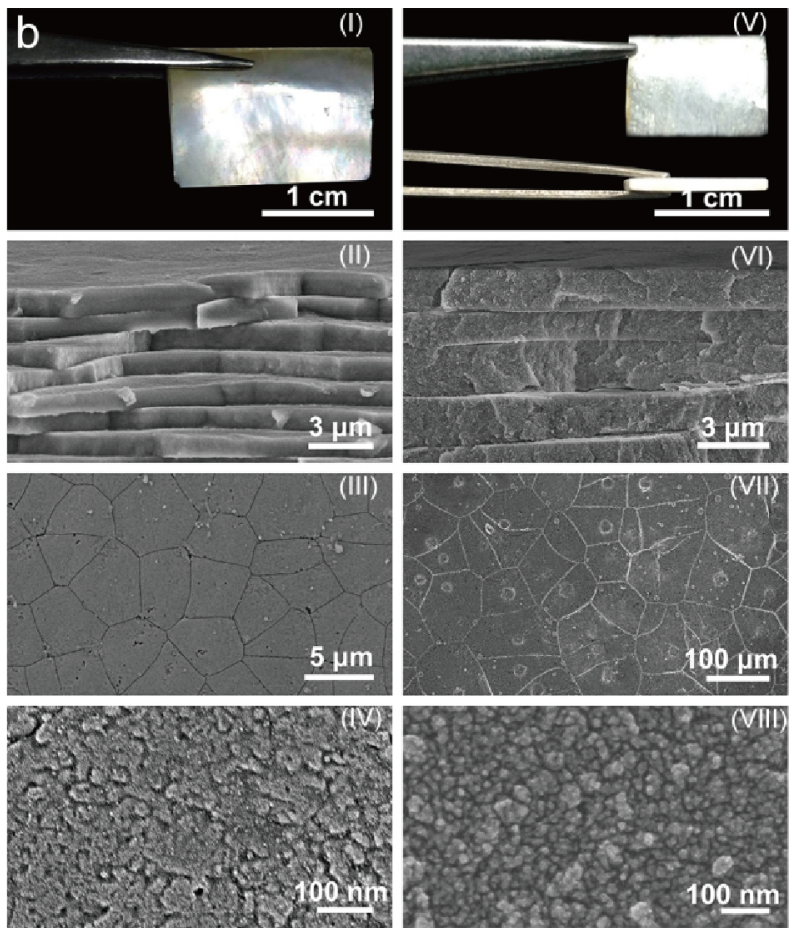

Figure 1 Synthetic nacre versus natural nacre. (a) Mineralization of the predesigned matrix. (b) Structural similarity of natural nacre and the synthetic nacre. (c) Rising crack resistance curve (R-curve) of the synthetic nacre, demonstrating the extrinsic toughening mechanism. Reprinted with permission from Ref. [12], Copyright 2016, American Association for the Advancement of Science.

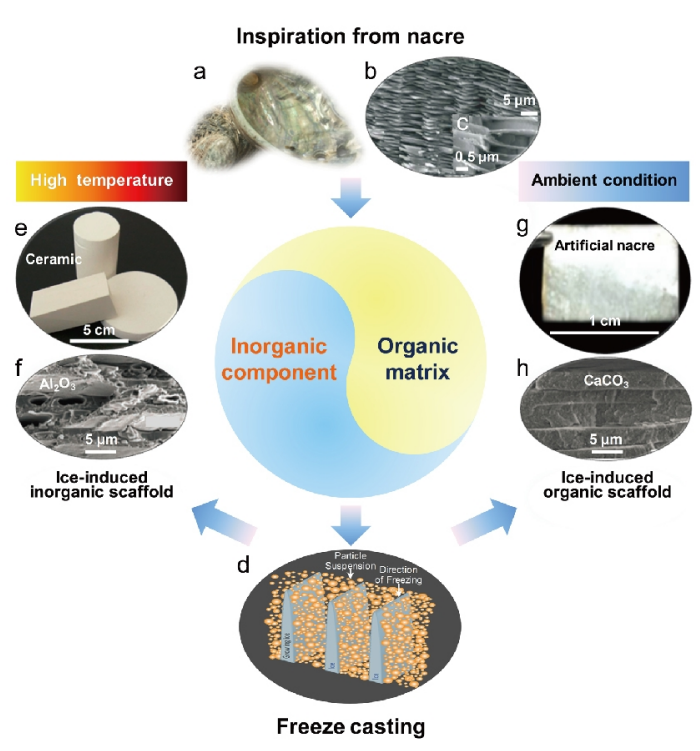

Figure 2 Freeze casting for mimicking natural nacre. (a) Digital image of natural nacre. (b and c) Brick-mortar hierarchical micro/nano-scale structure with inorganic component of calcium carbonate glued with organic protein matrix. The nacre is the typical binary cooperative complementary material system. Reprinted with permission from Ref. [7], Copyright 2006, American Association for the Advancement of Science. (d) The freeze casting for mimicking nacre is developed by Tomsia et al. Reprinted with permission from Ref. [3], Copyright 2014, Nature Publishing Group. (e and f) The freeze casting technique of ice-induced inorganic scaffold is utilized to fabricate high performance bulk ceramic composites with high temperature operation. Reprinted with permission from Ref. [8], Copyright 2008, American Association for the Advancement of Science. ( $g$ and $h$ ) The freeze casting technique of ice-induced organic scaffold is demonstrated for precisely mimicking mineralization of nacre at ambient condition. Reprinted with permission from Ref. [12], Copyright 2016, American Association for the Advancement of Science. 
Tomsia's group developed a novel freeze casting technique with ice-induced template for mimicking nacre to obtain high performance ceramic composites [7]. However, it needs high temperature to sinter inorganic precursor for preparing the layered scaffold (Figs 2e and f) [8], which limits its application for constructing heat-labile materials at ambient condition. Contrary to the ice-induced inorganic scaffold [7-10], Yu's group creatively developed ice-induced organic scaffold to precisely mimic the mineralization of nacre [12]. The synthetic nacre shows the highest similar structure and components like natural nacre until now (Figs $2 g$ and $h$ ) [12], and excellent mechanical properties comparable to natural nacre.

This work has been highlighted by Nature as entitled of "Bulk production of mother-of-pearl" [14], but more important breakthrough is mineralization under ambient conditions for mimicking nacre. As a significant breakthrough in the fabrication of bio-inspired materials, commented by the anonymous reviewers ("this is a solid and pioneering work", "this manuscript will contribute to the design principles for preparing robust composite materials with hierarchically ordered structures using various constituents including brittle and heat-labile building blocks, which is instructive for future work"), this innovative strategy is applicable to a wide range of components including ceramics and metal-organic frameworks (MOFs). In addition, by applying different predesigned matrix and mineralization/precipitation conditions, the nano- to microscopic structures can be controlled. This feasible strategy represents a rational strategy for the preparation of robust composite materials with hierarchically ordered structures, where various constituents are adaptable, including brittle and heat-labile materials, showing broad potential ap- plications in many fields such as tissue engineering and aerospace industry.

Received 22 August 2016; accepted 22 August 2016; published online 29 August 2016

1 Cheng Q, Jiang L, Tang Z. Bioinspired layered materials with superior mechanical performance. Acc Chem Res, 2014, 47: 1256-1266

2 Yao HB, Ge J, Mao LB, et al. 25th anniversary article: artificial carbonate nanocrystals and layered structural nanocomposites inspired by nacre: synthesis, fabrication and applications. Adv Mater, 2014, 26: 163-188

3 Wegst UGK, Bai H, Saiz E, et al. Bioinspired structural materials. Nat Mater, 2014, 14: 23-36

4 Tang Z, Kotov NA, Magonov S, et al. Nanostructured artificial nacre. Nat Mater, 2003, 2: 413-418

5 Podsiadlo P, Kaushik AK, Arruda EM, et al. Ultrastrong and stiff layered polymer nanocomposites. Science, 2007, 318: 80-83

6 Bonderer LJ, Studart AR, Gauckler LJ. Bioinspired design and assembly of platelet reinforced polymer films. Science, 2008, 319: 1069-1073

7 Deville S. Freezing as a path to build complex composites. Science, 2006, 311: 515-518

8 Munch E, Launey ME, Alsem DH, et al. Tough, bio-inspired hybrid materials. Science, 2008, 322: 1516-1520

9 Bouville F, Maire E, Meille S, et al. Strong, tough and stiff bioinspired ceramics from brittle constituents. Nat Mater, 2014, 13: 508-514

10 Bai H, Chen Y, Delattre B, et al. Bioinspired large-scale aligned porous materials assembled with dual temperature gradients. Sci Adv, 2015, 1: e1500849-e1500849

11 Le Ferrand H, Bouville F, Niebel TP, et al. Magnetically assisted slip casting of bioinspired heterogeneous composites. Nat Mater, 2015, 14: 1172-1179

12 Mao LB, Gao HL, Yao HB, et al. Synthetic nacre by predesigned matrix-directed mineralization. Science, 2016, 354: 107-110

13 Liu M, Jiang L. Dialectics of nature in materials science: binary cooperative complementary materials. Sci China Mater, 2016, 59: 239-246

14 Materials science: Bulk production of mother-of-pearl. Nature 2016, 536: 377 